\title{
MRI Surveillance of Plastic Material Surgical Meshes: Experimental Model - Interim Results
}

\author{
ADRIAN CARABINEANU ${ }^{1 \#, ~ R A M O N A ~ G A D E A ~}{ }^{1,2 \#}$, DAN COSTACHESCU ${ }^{3}$, \\ ADELINA MOCANU ${ }^{2}$, DAN NAVOLAN ${ }^{2}$, DANIEL MALITA ${ }^{3 *}$, RALUCA CEAUSU ${ }^{4 *}$, \\ CRISTINA SARACIN ${ }^{3}$, OCTAVIAN CRETU ${ }^{1}$, ALEXANDRU BLIDISEL ${ }^{1}$ \\ ${ }^{1}$ Victor Babes University of Medicine and Pharmacy of Timisoara, Faculty of Medicine, $1{ }^{\text {st }}$ Department of Surgery, 2 Eftimie \\ Murgu Sq., 300041, Timisoara, Romania \\ ${ }^{2}$ Victor Babes University of Medicine and Pharmacy of Timisoara, Faculty of Medicine, Department of Obstetrics- \\ Gynecology, 2 Eftimie Murgu Sq., 300041, Timisoara, Romania \\ ${ }^{3}$ Victor Babes University of Medicine and Pharmacy of Timisoara, Faculty of Medicine, Department of Radiology, 2 Eftimie \\ Murgu Sq., 300041, Timisoara, Romania \\ ${ }^{4}$ Victor Babes University of Medicine and Pharmacy of Timisoara, Faculty of Medicine, Department of Microscopic \\ Morphology/Hystology, 2 Eftimie Murgu Sq., 300041, Timisoara, Romania
}

\begin{abstract}
The number of surgical procedures for abdominal wall defects is increasing, often requiring the insertion of plastic material meshes. Surveillance of patients with inserted plastic meshes requires an accurate determination of the position of the mesh. However, this is a difficult task, depending on the kind of mesh, magnetic resonance imaging (MRI) protocol or consistence of the surrounding tissue (fat, muscle, aponeurosis). The aim of our research was to develop an experimental model to test the ability of MRI to identify the exact position of surgical plastic meshes: polypropylene or polyester. To simulate the placement of a mesh in human body we developed a model built up from two pieces of tissue with dimensions of $40 \mathrm{~cm} \times 20 \mathrm{~cm}$, harvested from a pig with a weight of $120 \mathrm{~kg}$. The meshes were situated for MRI evaluation between the two pieces: abdominal pig muscle respectively suprajacent abdominal pig wall subcutaneous fat, approximately $2 \mathrm{~cm}$ high. Five surgical meshes were scanned through six MRI sequences, in view of establishing an optimal MRI scanning protocol and best visible meshes. The MRI scans were evaluated by 5 radiologists with different degrees of training. Our results showed that the experimental model developed by us can be successfully used to test the ability of MRI to visualize different kind of plastic meshes. Also, our experiment has revealed that T1fl2D sequence is the best in highlighting meshes from surrounding tissue, and the best visualized Mesh was number 4, made of polyester. In conclusion, based on our experimental model, we should select a plastic mesh or MRI protocol which will allow an optimal post implantation monitoring. Modern technology of material's fabrication can help to better identify the mesh itself using MRI scanning.
\end{abstract}

Keywords: surgical mesh, MRI, abdominal wall defects, visualization, animal model

\section{Introduction}

Surgical repair of abdominal wall weakness or defects represents a common procedure which could be done with native tissue or synthetical meshes. The experience of surgeons, the strength and perfusion of affected tissues, particularities of patients or type of available meshes play a role in choosing one or the other of the repair options [1,2]. If the patient's native tissue has been compromised, meshes are most often necessary to supplement tissue strength. Nowadays, up to $75-80 \%$ of cases which require hernia surgery benefit from a plastic material mesh. The diversity of plastic meshes for surgical repair has stirred the interest of surgeons and radiologists in studying features of meshes: composition, structure, price, risk of infection, inflammatory response of surrounding tissue and imagistic monitoring of implanted meshes. Advantages and disadvantages of these plastic meshes ought to be recognized and understood before choosing procedure and materials.

*email: malita.daniel@umft.ro,ra.ceausu@umft.ro

\#Both authors equally contributed to this work and should be considered first author 
After an abdominal wall repair with surgical mesh, monitoring is necessary. Imaging (MRI or CT) of meshes can be difficult, which is why this actual problem has come to our attention. Imagistic follow up is necessary in order to find out the position of the mesh: to see if it slides from the primary position or to determine the margins in case of a reintervention. When this study was performed, companies that produce these meshes were not providing the practitioners any information regarding the radiopacity or radiolucency of the mesh $[3,4]$.

Most synthetic surgical meshes are made of two types of plastic materials: polyester (Figure 1) and polypropylene (Figure 2). Polyesters are polymers which contain repetitive esters structures [5]. The majority of synthetic polyesters are not biodegradable. Polyesters have good mechanical properties and could be used to sustain structures, including anatomic structures, such as the use of meshes for hernia repair. Polyesters are a category of polymers that contain an ester functional group in their main chain [5]. As a material, the term most often refers to polyethylene terephthalate. The synthesis of polyesters is generally done through a polycondensation reaction [5]. Polypropylene is a thermoplastic product produced by propylene polymerization [6]. Polypropylene is the most used substance in manufacturing synthetic surgical meshes used to repair anatomic defects and to prevent hernia and vaginal or genital prolapse. Polypropylene is well tolerated by human tissue and rejections occur very rarely. Polypropylene is a more heat-resistant plastic than polyvinyl chloride (PVC). Structurally, it is a vinyl polymer, and it is similar to polyethylene, only that every other carbon atom in the backbone chain has a methyl group attached to it [7].

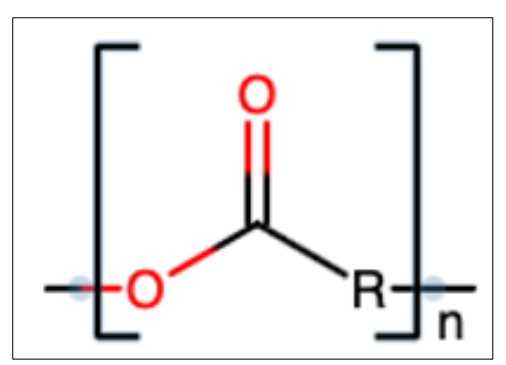

Figure 1. Structure of polyester

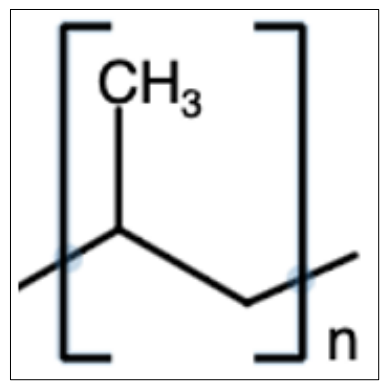

Figure 2. Structure of polypropylene

Newer experience showed that after mesh insertion in certain cases (vaginal wall) complications occur which could lead to new surgical procedures to remove the mesh [8]. Thus, Food and Drug Administration (FDA) ordered manufacturers and suppliers to stop selling meshes used to repair vaginal defect (cystocele) [8].

Polypropylene and polyester meshes used in hernia repair caused similar inflammatory responses.

After a systematic analysis of literature we found there are a significant number of case reports but a very few experimental studies, or in vivo studies, regarding the visualization of the surgical meshes with the help of MRI scanning. Taking the aforementioned into account, we aimed in our study to develop an experimental model to test the ability of MRI to identify the exact position of synthetic surgical meshes and to compare the visibility of different meshes according to their features: plastic materials composition (polypropylene or polyester) and texture [9].

\section{Materials and methods}

\subsection{Experimental model}

To simulate the placement of a mesh in the human body we developed a model built up from two pieces of tissue, with dimensions of $40 \mathrm{~cm} \times 20 \mathrm{~cm}$, harvested from a pig weighing $120 \mathrm{~kg}$, to ensure a layer of fat similar to that of the body of patients with this type of pathology. The meshes were situated for MRI evaluation between the two pieces: abdominal pig muscle respectively suprajacent abdominal pig wall subcutaneous fat with a height of approximately $2 \mathrm{~cm}$. 


\subsection{MRI evaluation of meshes}

We used a T Siemens Magnetom AERA MRI machine to perform the experiment. There were 6 scanning sequences: T2 TSE tra, T2 ME2 tra, T2 CISS3D tra, T2 SWI tra, T1 fl2D opp-in tra, T1 fl3D non fat sat tra. Five investigators (two consultants, two specialists and one resident) contributed to MRI evaluation of each mesh. Each investigator noted a score from 1 to 5 to evaluate the visibility of meshes and, respectively, their margins. Since our study was performed on an avital animal model, we only had the possibility to evaluate the presence of the mesh but not the inflammatory response it caused.

\subsection{Synthetic meshes}

In our experimental study we used five plastic meshes (M1->M5) from which two were made of polypropylene (Parietene and Surgipro) and three of polyester (ProGrip, Parietex and Symbotex) (Table $1)$.

Table 1. Characteristics of meshes used in our experiment

\begin{tabular}{|c|c|c|c|c|c|c|}
\hline $\begin{array}{l}\text { Mesh } \\
\text { number }\end{array}$ & $\begin{array}{c}\text { Commercial } \\
\text { name }\end{array}$ & Material & $\begin{array}{l}\text { Density } \\
\mathrm{g} / \mathrm{m}^{2}\end{array}$ & $\begin{array}{c}\text { Size } \\
(\mathrm{cm} / \mathrm{cm})\end{array}$ & $\begin{array}{l}\text { Porosity } \\
(\mathrm{mm} / \mathrm{mm})\end{array}$ & Visceral absorbable film \\
\hline M 1 & $\begin{array}{l}\text { Parietene } \\
\text { (Medronic) }\end{array}$ & $\begin{array}{l}\text { monofilament } \\
\text { polypropylene }\end{array}$ & 47 & $10 / 15$ & $2.0 / 2.4$ & No \\
\hline M 2 & $\begin{array}{c}\text { Surgipro } \\
\text { (Covidien) }\end{array}$ & $\begin{array}{l}\text { monofilament } \\
\text { polypropylene }\end{array}$ & 42 & $15 / 15$ & $0.7 / 1.0$ & No \\
\hline M 3 & $\begin{array}{c}\text { ProGrip } \\
\text { (Medronic) }\end{array}$ & $\begin{array}{c}\text { semiabsorbable } \\
\text { monofilament } \\
\text { polyester }\end{array}$ & 84 & $10 / 15$ & $1.8 / 1.8$ & glycerol $30 \%$ and collagen $70 \%$ \\
\hline M 4 & $\begin{array}{l}\text { Parietex } \\
\text { (Covidien) }\end{array}$ & polyester composite & 79 & $15 / 20$ & $1.5 / 1.8$ & $\begin{array}{l}65 \% \text { bovine collagen, } 22 \% \\
\text { polyethylene glycol and } 13 \% \\
\text { glycerol }\end{array}$ \\
\hline M 5 & $\begin{array}{l}\text { Symbotex } \\
\text { (Covidien) }\end{array}$ & $\begin{array}{c}\text { monofilament } \\
\text { polyester }\end{array}$ & 66 & $15 / 20$ & $2.3 / 3.3$ & $\begin{array}{l}65 \% \text { bovine collagen, } 22 \% \\
\text { polyethylene glycol and } 13 \% \\
\text { glycerol }\end{array}$ \\
\hline
\end{tabular}

2.3.1.1. PPM Macroporous (Parietene) (Medtronic) (M 1) is a 2D macroporous mesh made from monofilament polypropylene used in therapy of ventral and inguinal hernias. It can be remodeled without deformation risk, has a density at implantation of $47 \mathrm{~g} / \mathrm{m}^{2}$, the porous dimension of $2.0 \times 2.4 \mathrm{~mm}$ and dimensions of $10 \mathrm{~cm} \times 15 \mathrm{~cm}$. Polypropylene is obtained by polymerization in the presence of triethylaluminium and titanium tetrachloride of propylene. Polypropylene has better abrasion resistance and chemical agents and good electrical insulating properties. Due to its thermoplastic properties, it is used to obtain fibers and films resistant to breaking, bending and shearing.

2.3.1.2. Surgipro (Covidien) (M 2) is a $2 \mathrm{D}$ mesh from monofilament propylene which is used to repair ventral and inguinal hernias. It can be modeled without deformation risk with density of $42 \mathrm{~g} / \mathrm{m}^{2}$ and mesh dimensions of $15 \mathrm{~cm} \times 15 \mathrm{~cm}$.

2.3.1.3. ProGrip (Self Gripping Polypropylene Mesh) (Medtronic) (M 3) is a surgical mesh precut, made from semi absorbable monofilament polyester which facilitates fast integration in the implantation site, and it is used in the laparoscopic and laparotomic treatment of inguinal hernias. The mesh is provided with $1 \mathrm{~mm}$-sized polylactic acid legs that have a self-fixing role without the need for other devices or materials. This fastening system allows uniform attachment of the net without tension and provides increased comfort to the patient. This mesh presents an absorbable film from glycerol $30 \%$ and collagen $70 \%$, which facilitates the manipulation of the mesh, being absorbed within $24 \mathrm{~h}$ from the moment of implantation. The density of the mesh is $84 \mathrm{~g} / \mathrm{m}^{2}$, with porous dimensions of $1.8 \mathrm{~mm} \times 1.8$ $\mathrm{mm}$. After the absorption of the legs, the density is reduced to $49 \mathrm{~g} / \mathrm{m}^{2}$. The absorption of the legs is completed 15 months from the implantation. For better anatomical orientation, this mesh is fitted with a green medial marking [10,11]. 
2.3.1.4. Parietex (Covidien) (M 4) is a surgical mesh made of polyester composite, in the shape of a right-angle triangle and it is used in the treatment of small and medium size ventral hernias. These resistant fibers have a lot of uses. The parietal side is made of monofilament polyester with a 3D structure, with pore size of $1.5 \mathrm{~mm}$ x $1.8 \mathrm{~mm}$. It presents a colored monofilament polyester component with a 2D structure for better visualization, with 4 cardinal fixing points and pore size of $1.1 \mathrm{~mm} \times 1.6$ $\mathrm{mm}$. The visceral side has an absorbable film made of $65 \%$ bovine collagen, $22 \%$ polyethylene glycol and $13 \%$ glycerol with absorption in maximum 4 weeks.

2.3.1.5. Symbotex (Covidien) (M 5) is a round surgical mesh used in the laparoscopic and laparotomic surgical treatment of ventral and inguinal hernias. It is a double side mesh made out of monofilament polyester with best positioning preperitoneal. The parietal side had a 3D monofilament polyester structure with pore size of $2.3 \mathrm{~mm} \times 3.3 \mathrm{~mm}$, mesh thickness of $0.6 \mathrm{~mm}$ and density of $66 \mathrm{~g} / \mathrm{m}^{2}$. It presents a $2 \mathrm{D}$ blue central mark for better anatomical orientation. The visceral side has an absorbable film made of $65 \%$ bovine collagen, $22 \%$ polyethylene glycol and $13 \%$ glycerol with absorption in maximum 4 weeks. Also, it has the properties of a temporary adhesive, facilitating placement and fixation.

\subsection{MRI evaluation of meshes}

The visibility of a surgical mesh is defined as the property to be seen through imagistic techniques of high resolution. The meshes are visible either directly or indirectly by the inflammatory reaction they produce. The visibility of the meshes used in this experiment was analyzed by a team of five medical radiologists, namely two primary radiologists with over 10 years of experience, two medical radiologists with more than five years of experience and one resident in radiology with a three-year experience. A score from 1 to 5 was given by each radiologist for the evaluation of the presence, respectively of the edges of meshes for each MRI sequence. A similar evaluation method performed by radiologists with different professional experience was also used in other studies [12].

\subsection{Statistical issues}

We stored data in Microsoft Office Excel (Microsoft Corporation. Redmond, Washington, USA). We used Instat GraphPad Prism Software (GraphPad Software, Northside Dr. Suite 560, San Diego, CA, USA) for statistical analysis. Since the variables were non-Gaussian distributed the results are presented as medians and [interquartile ranges]. To assess the significance of the differences between three or more groups, Kruskal-Wallis test corrected for ties was used. Dunn's multiple comparisons test was used to compare the difference in the sum of ranks between two columns.

\section{Results and discussions}

\subsection{Overall visibility of the five plastic meshes}

The visibility (of the presence and edges) of each mesh (M1-M5) was evaluated. Each mesh received two scores (from 1 to 5) from each examinator, one for the visibility of the presence, respectively another for the visibility of edges. The visibility evaluation was repeated for each of the six MRI sequences. A median value was calculated from the 60 visualization scores (scores from 1 to 5) obtained by each of the five physicians on evaluating (the presence and the edges) of each mesh in all the six different MRI sequences. For the visualization of each mesh were obtained sixty values: thirty for the presence, respectively thirty for the edges of each mesh. A very significant difference $(\mathrm{p}=0.006)$ was found regarding the visibility of the six types of meshes (Table 2). Mesh 4 was better visualized compared to Mesh $1(\mathrm{P}<0.05)$, respectively Mesh $2(\mathrm{p}<0.05)$. Also, Mesh 4 was better visualized than Mesh 3, respectively Mesh 5, but the difference did not reach a significant value. Our results showed that the Mesh 4 had the best visibility followed by the mesh number 3 and number 5, while the mesh 2 and mesh 1 had the worst visibility in our model. We noticed that mesh 4 and 5 are made of polyester, while mesh 3 is made of polypropylene. The natural question is whether the difference in visibility is due to the 
material or the way the mesh is built. Our experimental model is very easy to repeat and also cheap, but it has some limitations, namely that it does not capture the body's reaction to the mesh. The visibility of the mesh in the conditions of the body's fibrosis reaction to the mesh can be evaluated only in a living tissue. So, we believe that the reason for the difference in visibility is the way the mesh is made and not the composition of the material.

Table 2. Median of meshes visibility scores by five radiologists in the six MRI sequences. A score from 1 to 5 was given by each radiologist $(n=5)$ for the evaluation of the presence, respectively of the edges of meshes for each MRI sequences $(n=6)$. A total of 60 scores/mesh

\begin{tabular}{|c|c|c|c|c|c|c|}
\hline all MRI Sequences & Mesh 1 & Mesh 2 & Mesh 3 & Mesh 4 & Mesh 5 & P value \\
\hline value Median & 2 & 2.5 & 3 & 3.5 & 3 & 0.006 \\
[interquartile range] & {$[2.75]$} & {$[3]$} & {$[4]$} & {$[3]$} & {$[2.75]$} & \\
\hline
\end{tabular}

\subsection{MRI sequences and visibility of meshes}

Each mesh was evaluated in six MRI sequences. Each of the five radiologists appreciated the presence of the mesh, the edges of the meshes, giving two scores (from 1 to 5) for each mesh, one score for the evaluation of the body and one score for the evaluation of the edges of the mesh. Thus, for each mesh and MRI sequence ten evaluation scores were given. The ability of MRI sequences to visualize each meshes was compared (Table 3). Significant differences between the ability of MRI sequences to visualize meshes were found (Table 3). T1fl2D opp-in sequence showed the best ability to visualize meshes followed well behind by T2ME2 and T2SWI sequences.

Table 3. Median [interquartile range] of visibility scores for each mesh and MRI sequence evaluated by the five radiologists. For each mesh evaluated by an MRI sequence, two scores (from 1 to 5) were given by each radiologist: first for evaluation of the presence, respectively a second one for evaluation of the edges of meshes. In total 10 scores / mesh / MRI sequence

\begin{tabular}{|c|c|c|c|c|c|c|c|}
\hline $\begin{array}{c}\text { Median } \\
\text { (interquartile range) }\end{array}$ & T2TSE & T2ME2 & T2CISS3D & T2SWI & $\begin{array}{l}\text { T1fl2D } \\
\text { opp-in }\end{array}$ & T1fl3D & $P$ value \\
\hline Mesh 1 & $\begin{array}{c}1 \\
{[0]}\end{array}$ & $\begin{array}{l}2.5 \\
{[2]}\end{array}$ & $\begin{array}{l}1.5 \\
{[2]}\end{array}$ & $\begin{array}{c}3 \\
{[4]}\end{array}$ & $\begin{array}{c}4 \\
{[1]}\end{array}$ & $\begin{array}{l}1.5 \\
{[1]}\end{array}$ & $<0.0001$ \\
\hline Mesh 2 & $\begin{array}{c}1 \\
{[0]}\end{array}$ & $\begin{array}{l}2.5 \\
{[2]}\end{array}$ & $\begin{array}{l}2.5 \\
{[2]}\end{array}$ & $\begin{array}{c}3 \\
{[3.75]}\end{array}$ & $\begin{array}{c}4 \\
{[1]}\end{array}$ & $\begin{array}{c}2 \\
{[1]}\end{array}$ & $<0.0001$ \\
\hline Mesh 3 & $\begin{array}{c}1 \\
{[1]}\end{array}$ & $\begin{array}{l}4.5 \\
{[1]}\end{array}$ & $\begin{array}{c}3 \\
{[0.25]}\end{array}$ & $\begin{array}{c}3 \\
{[2]}\end{array}$ & $\begin{array}{c}5 \\
{[0]}\end{array}$ & $\begin{array}{c}1.5 \\
{[1.5]}\end{array}$ & $<0.0001$ \\
\hline Mesh 4 & $\begin{array}{c}2 \\
{[2]}\end{array}$ & $\begin{array}{l}3.5 \\
{[3]}\end{array}$ & $\begin{array}{c}5 \\
{[0]}\end{array}$ & $\begin{array}{l}3.5 \\
{[4]}\end{array}$ & $\begin{array}{c}5 \\
{[0]}\end{array}$ & $\begin{array}{c}2 \\
{[2]}\end{array}$ & $<0.0001$ \\
\hline Mesh 5 & $\begin{array}{c}1 \\
{[1]}\end{array}$ & $\begin{array}{c}2.5 \\
{[3.25]}\end{array}$ & $\begin{array}{l}2.5 \\
{[2]}\end{array}$ & $\begin{array}{c}2.5 \\
{[2.25]}\end{array}$ & $\begin{array}{c}4 \\
{[1]}\end{array}$ & $\begin{array}{c}3 \\
{[0.25]}\end{array}$ & $<0.0001$ \\
\hline Total & 6 & 15.5 & 14.5 & 15 & 22 & 10 & \\
\hline
\end{tabular}

Taken together the results above showed that the best sequence to evaluate meshes is T1fl2D oppin, followed T2ME2, T2SWI and T2CISS3D, whereas Mesh 3 and Mesh 4 showed the best visibility compared to other ones.

The T1 fl2D opp-in tra sequence ensures the best visibility both for identifying the position of the surgical mesh and for highlighting its edges, regardless of the type of mesh used. The identification of the position of the mesh is superior to the identification of its edges. The T1 fl2D opp-in tra is a chemicalshift sequence, and, basically, the meshes are identified indirectly secondary to the chemical shift 
artefact. Chemical shift is secondary to differences between resonance frequencies of fat and water. It occurs in the frequency-encode direction where a shift in the detected anatomy occurs because fat resonates at a slightly lower frequency than water [13].

T2 CISS 3D was another good sequence for mesh visualization; being a 3D sequence, thinner slices were acquired making the spatial resolution being superior. Similar results were obtained by Chen $L$ et al [14] with a similar 3D sequence on a polypropylene mesh.

T2 SWI is an MRI sequence that is extremely sensitive to compounds which distort the magnetic field; most of the plastics, including polypropylene are diamagnetic [15]. Compounds that have paramagnetic, diamagnetic or ferromagnetic properties interact with the magnetic field thus distorting it and altering the phase of local tissue, resulting in loss of signal [16,17]. This signal loss results in the indirect visualization of the mesh.

The T2 TSE sequence along with T2CISS3D are the only used sequence in our study which doesn't produce any intrinsic artefacts together with the polypropylene meshes and also it is not a 3D sequence thus resulting in a low susceptibility in mesh visualization. Basically, the best sequences for mesh visualization used in our study have 2 important characteristics: they are either 3D, which provides a good spatial resolution, or they produce different artefacts which indirectly allow the radiologist to identify the position of the mesh.

Our study shows us which of the 5 meshes is best viewed and what type of sequence should be used to evaluate them. The results of our study encourage us to perform a study in living tissue to evaluate the mesh in the conditions of the body's reaction to it and the fibrosis tissue. It is difficult to predict whether studies in the live animal model will confirm this ranking of table visibility [18].

\section{Strength and weakness of study}

The strength of our study is that the experiment is cheap, repeatable and easy to be performed allowing comparison of different MRI protocols and the visibility of different meshes. The weakness of our study is that an experiment performed in nonviable tissue does not allow the study of the reaction of the surrounding tissue and the visibility of meshes after the granular reaction appears.

Categorizing the surgical meshes by visibility should include the MRI sequence where the mesh is graded. Within the limitations (small sample, lack of in vivo reaction) of the study we can conclude the following:

The analysis of the results of the experimental study shows that the visibility of surgical nets differs depending on the MRI sequence and the type of net. There are some nets that can be viewed on one or more MRI sequences. Visibility through these high-resolution imaging techniques is determined by several factors: thickness, density, size of the pores, the existence of an absorbable film on one side of the net, but also by the type of material that triggers the patient's inflammatory reaction. Thickness of the mesh is important because the visibility of thicker meshes was better than the thinner ones. Meshes with absorbable films from one side or impregnated (Parietex and Symbotex) are thick. Gripping surgical mesh (Progrip) had a better visibility by the presence of grips. If the density of the material is similar to the one of the surrounding tissues (fat tissue and muscle), the visibility is lower. The ones with big density implanted in the animal carcass (more than $50 \mathrm{~g} / \mathrm{m}^{2}$ ) exceeded better visibility (Parietex, Symbotex and Progrip). The structure of the surgical mesh is another criterion of its visibility in MRI, depending on whether they are woven or knitted. Any surgical mesh triggers an inflammatory response, and it follows the healing stages of any other type or reactive inflammation (inflammation, angiogenesis, fibroplasia, matrix filling and contraction), which ultimately leads to the final deposition of collagen and the connective tissue formation. Thus, rigid scar plates are formed especially in polypropylene mesh, which are also felt on palpation. This aspect could not be observed in this study since it was performed on animal carcasses. On an MRI scan subtle modifications regarding the inflammatory response of the surrounding tissue can be detected, showing the indirect visibility of the mesh. These modifications are very weak, and they require the radiologists' close attention to the patient's history which mostly goes unnoticed [18-20]. 
Surgical meshes can be classified in groups according to their visibility in MRI. The classification of meshes that the authors want to propose is one that helps the surgeon and the radiologist to understand the properties of the meshes that are going to be implanted, or the ones that must be identified radiologically. All of the surgical meshes have to be labeled as visible, faintly visible, invisible and indirectly identified, and the identification or non-identification of the mesh' margins must also be mentioned [21].

Visible meshes have a homogenous structure and a thickness more than $1 \mathrm{~mm}$ and this allows a very good measuring on the whole surface. Meshes made of polyester are more visible and they have an absorbable film from collagen, glycerol and polyethylene glycol, at the moment of implantation. Meshes of polypropylene have a faint visibility, they do not allow good measuring and their margins cannot be seen. MRI identifies the inflammatory response of the indirectly identified mesh, which in this study was missing since the specimens were carcasses. Invisible meshes are those that do not bring any information on the MRI [21,22].

Surgical meshes need to be visible for a better understanding of potential complications that result from the surgical intervention of hernia, classic or laparoscopic, such as relapse, swelling of the mesh and contraction of the mesh, as well as to help the surgeon to position the initial incision at the right place. Therefore, manufacturers and surgeons ought to come up with a method of impregnating a marker at the surface of the surgical mesh, to make it more visible [23]. The authors believe that a software should be created that could identify different densities given by different meshes more easily.

\section{Conclusions}

Our results showed that the experimental model developed by us can be successfully used to test the ability of MRI to visualize different kind of plastic meshes. Also, our experiment has revealed that T1fl2D sequence is the best in highlighting meshes from surrounding tissue, and the best visualized mesh was number 4 (M4-Parietex), made of polyester. In conclusion, based on our experimental model, the selection of a plastic mesh or MRI protocol would allow an optimal post implantation monitoring.

\section{References}

1.FITZGERALD, J.F., KUMAR, A.S., Biologic versus Synthetic Mesh Reinforcement: What are the Pros and Cons? Clin Colon Rectal Surg. 27(4), 2014, 140-148

2.BITTNER, R., BINGENER-CASEY, J., DIETZ, U., FABIAN, M., FERZLI, G.S., FORTELNY, R.H., KÖCKERLING, F., KUKLETA, J., LEBLANC, K., LOMANTO, D., MISRA, M.C., BANSAL, V.K., MORALES-CONDE, S., RAMSHAW, B., REINPOLD, W., RIM, S., ROHR, M., SCHRITTWIESER, R., SIMON, SMIETANSKI, TH.M., STECHEMESSER, B., TIMONEY, M., CHOWBEY, P., Guidelines for laparoscopic treatment of ventral and incisional abdominal wall hernias (International Endohernia Society (IEHS)- part 1), Surg. Endosc., 28(1), 2014, 2-29.

3.GERNOT FITZ GERALD, J.F., KUMAR, A.S., Biologic versus Synthetic Mesh Reinforcement: What are the Pros and Cons? Clin. Colon. Rectal. Surg. 27(4), 2014, 140-148.

4.BULBULLER, N., KIRKIL, C., GODEKMERDAN, A., AYGEN, E., ILHAN, Y.S., The comparison of inflammatory responses and clinical results after groin hernia repair using polypropylene or polyester meshes, Indian J. Surg., 77(Suppl2), 2015, 283-287.

5.KÖPNICK, H., SCHMIDT, M., BRÜGGING, W., RÜTER, J., KAMINSKY, W., Polyesters, Ullmann's Encyclopedia of Industrial Chemistry, Wiley-VCH Verlag., 2000.

6.GAHLEITNER, M., PAULIK, C., Polypropylene, Ullmann's Encyclopedia of Industrial Chemistry. Weinheim: Wiley-VCH. Verlag, 2014, 1-44.

7.***https://pediaa.com/difference-between-polyester-and-polypropylene/Difference Between poly-ester and polypropylene, November 2, 2017

8.FDA-Statement about Urogynecologic Surgical Mesh Implants, 16 April 2019, https://www.fda.gov/medical-devices/implants-and-prosthetics/urogynecologic-surgical-meshimplants (last seen February 2021). 
9.RAKIC, S., LEBLANC, K.A., The radiologic appearance of prosthetic materials used in hernia repair and a recommended classification, A.J.R., 201, 2013, 1180-1183.

10.KOHLER, G., PALLWEIN-PRETTNER, L., KOCH, O.O., LUKETINA, R.R., LECHNER M., EMMANUEL K., Magnetic resonance-visible meshes for laparoscopic ventral hernia repair JSLS.;19(1), 2015:e2014.00175.

11.KRAEMER, N.A., DONKER, H.C.W., OTTO, J., HODENIUS, M., SENEGAS, J., SLABU, I., KLINGE, U., BAUMANN, M., MUELLEN, A., OBOLENSKI, B., GUEMNTHER R.W., KROMBACH, G.A., A concept for magnetic resonance visualization of surgical textile implants. Invest. Radiol. 45(8), 2010, 477-483.

12.HANSEN, N.L., CIRITSIS, A., OTTO, J., BUSCH, D., KUHL, C.K., KRAEMER N.A., Utility of magnetic resonance imaging to monitor surgical meshes, Investigative Radiology, 50(7), 2015, 436-442. 13.REISER, M.F., Magnetic Resonance Tomography. Springer Verlag, 2007, ISBN 354029354X.

14.CHEN, L., LENZ, F., ALT, C.D., SOHN, C., DE LANCEY, J.O., BROCKER, K.A., MRI visible Fe304 polypropylene mesh: 3D reconstruction of spatial relation to bony pelvis and neurovascular structures. Int Urogynecol J., 28(8), 2017, 1131-1138.

15.UEDA, Y., MISHIMA, F., AKIYAMA, Y., NISHIJIMA, S., Fundamental Study of Plastic Separation Utilizing Magnetic Force, in IEEE Transactions on Applied Superconductivity, 24(3), 2014, $1-5$.

16.TONG, K.A., ASHWAL, S., OBENAUS, A., NICKERSON, J.P., KIDO, D., HAACKE, E.M., Susceptibility-weighted MR imaging: a review of clinical applications in children. AJNR Am $J$ Neuroradiol.29(1), 2008, 9-17.

17.KUEHNERT, N., KRAEMER, N.A., OTTO. J.N, DONKER, H.C.W., SLABU, I., BAUMANN, M., KUHL, C.K, KLINGE, U., In vivo MRI visualization of mesh shrinkage using surgical implants loaded with superparamagnetic iron oxides. Surg. Endosc. 26(5), 2012, 468-1475.

18.HERNIASURGE GROUP, International guidelines for groin hernia Hernia Feb 22(1), 2018:1-165. 19.OZVERI E., SANLI DET, YILDIRIM D, GOK H, ERTEM M., Magnetic resonance visualization of iron-loaded meshes in patiens with pain after inguinal hernia repair. Hernia, 25, 2021, 727-732.

20.LECHNER, M., MEISSNITZER, M., BORHANIAN, K., BITTNER, R., KAUFMANN, R., MAYER, F., JAEGER, T, MITTERWALLNER, S., EMMANUEL, K, FORSTER. R., Surgical and radiological behavior of MRI -depictable mesh implants after TAPP repair: the IRONMAN study, Hernia 23(6), 2019,1133-1140.

21.FISCHER, T., LADURNER, R., GANGKOFER, A., MUSSACK, T., REISER, M., LIENEMANN, A., Functional cine MRI of the abdomen for the assessment of implanted synthetic mesh in patients after incisional hernia repair: initial results. Eur. Radiol. 17(12), 2007, 3123-3129.

22.LANGENBACH MR, SCHMIDT J, ZIRNGIBL H Comparison of biomaterials in the early postoperative period. Polypropylene meshes in laparoscopic inguinal hernia repair. Surg Endosc 17, 2003,1105-1109.

23.MUYSOMS, F., BECKERS, R., KYLE-LEINHASE, I., Prospective cohort study on mesh shrinkage measured with MRI after laparoscopic ventral hernia repair with an intraperitoneal iron oxide-loaded PVDF mesh. Surg. Endosc. 32(6), 2018, 2822-2830.

Manuscript received: 30.07 .2021 